\title{
ASSESSMENT OF SURFACE PARAMETERS OF MACHINE PARTS
}

\author{
Oskars Linins, Irina Boiko, Armands Leitans, Janis Lungevics \\ Riga Technical University, Latvia \\ oskars.linins@rtu.lv, irina.boiko@rtu.lv, armands.leitans@rtu.lv, janis.lungevics@rtu.lv
}

\begin{abstract}
The research paper describes 3D texture parameter use for potential wear resistance calculations for machined parts. The quality of the machined part surface is one of the most significant aspects, which influence their functional properties, including wear resistance. The wear process for two mating parts is unavoidable, and ultimately, it results in system failure. Low wear resistance reduces the lifetime of machine components, which leads to considerable material losses, ineffective use of resources, and in extreme cases, could even lead to a significant disaster. Therefore, researches devoted to the machine part functional parameter improvement, in particular by using various surface coatings, are fundamental. The development of highly functional surface coatings includes surface quality control using the appropriate tools and characterization technique. Widely used 2D profilometry technique does not provide relevant information on the actual surface condition; thus, one must develop a better method. Use of 3D texture parameter combination instead of typical 2D parameters can noticeably improve surface characterization precision, but from more than thirty 3D parameters, only as few as 2-8 parameters suit for wear prediction calculations. This research paper offers the potentially best set of the most appropriate 3D-texture parameters for assessing the functional surfaces of machine parts.
\end{abstract}

Keywords: texture, 3D-texture parameters, functional surfaces, tribology.

\section{Introduction}

The state and quality of the surface of the machine part is one of the most significant aspects, which influence their functional properties. Thus, a well-known wear phenomenon appears unavoidable and ultimately results in system failure. Besides, wear reduces the lifetime of machine components, which leads to considerable material losses, ineffective use of resources, and can result even to disastrous effects. The wear of the surfaces (including surfaces with coatings and nanocoatings, widely used to the improvement of functional parameters of the machine parts) is a complex process, which is characterized and affected by a variety of parameters such as the geometry of surface (roughness, texture, form deviation, etc.), physical and mechanical properties of the material, wear temperature and other conditions. Due to the complexity of the wear process, it is not possible to take into consideration all the influencing parameters. Therefore, wear calculation development, over time, developed in several directions [1]. Each direction is based on theoretical calculations taking into account assessing sets of affecting parameters. Nevertheless, there is still a problem in characterization of the functional surfaces, in particular in assessment of the surface parameters of the machine parts.

Widely used 2D roughness parameters do not provide appropriate information on actual (spatial) behaviour of the 3D objects [2-4], such as machine parts. Therefore, 3D surface parameters for evaluation of the performance of the machine parts could be the answer. The only question is, which of those 3D parameters will allow more precisely to predict the service life of products. For instance, in paper [5], the authors propose to use a special methodology for selecting the most relevant roughness parameters for a surface. It says that due to an increasing amount of new standardized parameters, the selection of actually usable parameters is a problem.

Additionally, at the moment, there is no single assessment of the importance of the parameters. Previously only a few researchers have tried to connect the quality parameters of spatial (3D) surface with the part functionality. Paper [6] presents a study on surface roughness, which was evaluated using $2 \mathrm{D}$ and $3 \mathrm{D}$ parameters. The results indicate that compared to $2 \mathrm{D}$ parameters ( $R a$ and $R z), 3 \mathrm{D}$ parameters $(S a$ and $S q)$ are more accurate to describe the surface quality. The standard error of 3D parameters is smaller than that of 2D. The work [7] shows the use of focus-variation microscopy to acquire 3D surfaces and analytically evaluate the surface quality of the laser cut edges using areal surface roughness parameters (parameters $S a$ and $S_{10 z}$ ). After analysis of the achieved results, it was concluded that the reliability of the standard 2D roughness measurements is low. Also, the research [8] stated that 3D parameters describe the processed surfaces more precisely than 2D parameters could. The following five 3D surface parameters were chosen in the research: Smmr, Sds, Sdq, Ssc and $S d r$. Research [9] proved that 2D roughness parameter $R a$ alone is inadequate for correlating with fatigue performance. In contrast, 3D surface texture parameters allowed to represent the surface and were 
recommended to use for topographic characterization, which can afterwards be used for correlation with fatigue performance prediction.

Only a few studies have investigated the correlation between surface 3D topography and tribology properties. Research [10] is devoted to the critical analysis of the description of the friction pair rough surface. It describes the need for evolution from 2D to 3D parameters. It was concluded that texture analysis could be efficiently applied for solving practical tribological problems in micro/nanoscale. Research [11] points out that the basic amplitude parameters that are commonly used to describe tribological characteristics are not enough to determine the tribological properties of contact surfaces. Therefore, other surface topography parameters should be used to describe the tribological properties of the friction pairs. It is crucial, because, according to [10], the initial surface topography has a significant influence on friction and wear under dry sliding conditions. Significant correlations were identified between several surface topography parameters and the wear volume: $S s k, S k$, and $S d q$. A modeling algorithm for predicting the surface roughness parameters $S a, S q$, Ssk, and $S k u$ under abrasive wear conditions was proposed by the authors of the work [12].

In research [13] it was found that from 4 surface texture parameters ( $S a, S s k, S d s$, and $S d q$ ), the $S a$ is most useful for characterizing surfaces that slide on the ice. In research [14] surface topographies, produced by different machining operations, were analyzed regarding their potential functionality. Parameters $S a, S s k, S p k, S d f, V m p$, and $V v v$ showed a close correlation with fluid retention abilities and tribological properties. Thus, from more than 30 standardized parameters [15] in the abovementioned researches [3-14], only 2-8 parameters were considered useful. The literature review suggests that 3D parameters may have a closer link with friction and wear characteristics than traditional 2D parameters; thus, researchers must carefully research which set of parameters is the most suitable for tribology calculations and property predictions.

Furthermore, according to the international survey on the use of surface texture parameters in the industry (179 responses from a total of 34 countries) [16], an increase in the range of surface texture parameters used, compared to the results from a survey done back in 1999, is observed. But still, only $30 \%$ of companies use new areal surface texture parameters. Some industry "sectors, for instance, are comprised of only a few participants, such as "tribology" $(2 \%)$... and thus are poorly represented".

This paper provides a a specific set of 3D-texture parameters, which could be used for assessing the functional surfaces of the machine parts, including ones with nanocoatings.

\section{D-texture parameters which describe the functional surfaces}

The following 3D-texture parameters [15] were measured and analyzed in [17] for defining the surfaces of the tribological parts: 1) amplitude parameters: $S a$, Ssk; 2) spatial parameter: Str, 3) functional (material volume) parameter: $V m c$, and 4) hybrid parameter: $S f d$. These 3D-texture parameters are significant for friction surfaces of the tribological parts because of their essence:

- $S a$ - arithmetic mean of the deviations from the mean plane, represents an overall measure of the texture comprising the surface;

- $S s k-$ skewness of the scale-limited surface. This parameter represents the degree of symmetry of the surface heights about the mean plane;

- $S t r$ - texture aspect ratio of the surface. This parameter measures the isotropy of the surface. This parameter has a result between 0 and 1 . If the value is near 1 , we can say that the surface is isotropic, i.e., it has the same characteristics in all directions. If the value is near 0 , the surface is anisotropic, i.e., has an oriented structure;

- $V m c$ - core material volume of the scale-limited surface; indicates a measure of the material forming the surface between various heights;

- $\quad S f d$ - fractal dimension of the surface (complexity of the surface), the smaller value specifies the more periodical surface (different from the random surface).

The described parameters can be used for any hard material surface characterization, which faces friction and wear. Functional coatings that are applied to relatively cheap bulk materials are becoming increasingly popular in the tribology field; thus, the further article will focus on functional coating surface characterization using five selected parameters. Good and bad texture examples will be discussed. 


\section{Examples and discussion}

This chapter includes different examples of a comparison of uncoated and coated surfaces from previously conducted researches. Their 3D texture images and parameters are shown, and comments about they tribology properties are discussed as well.

Firstly, the example of the sampes made from alloy steel Incoloy $800(\mathrm{Cr}-10.5 \ldots 12.0 \%$; $\mathrm{C}-$ $0.10 \ldots 0.16 \% ; \mathrm{Cu} \leq 0.30 \% ; \mathrm{Mn} \leq 0.60 \% ; \mathrm{Mo}-0.35 \ldots 0.50 \% ; \mathrm{Ni}-1.5 \ldots 1.8 \% ; \mathrm{P} \leq 0.30 \%$; $\mathrm{S} \leq 0.025 \% ; \mathrm{Si} \leq 0.60 \% ; \mathrm{V}-0.18 \ldots 0.30 \%$; W $-1.60 \ldots 2.0 \%)$ without coating and with 2-layer TiTiN PVD coating is shown. The images of 3D surfaces of samples without coating and with 2-layer Ti-TiN PVD coating are shown in Figure 1 and the 3D parameter values are given in Table 1. The materials and methods used in details are given in [17].

Secondly, the example of pure Copper $(\mathrm{Cu})$ coating and Carbon-Coper $(\mathrm{a}-\mathrm{CuC})$ coating, both applied to the same 100Cr6 steel substrate, are shown in Figure 2. For comparison, also the $100 \mathrm{Cr} 6$ substrate parameter values are shown in Table 2 .

a)

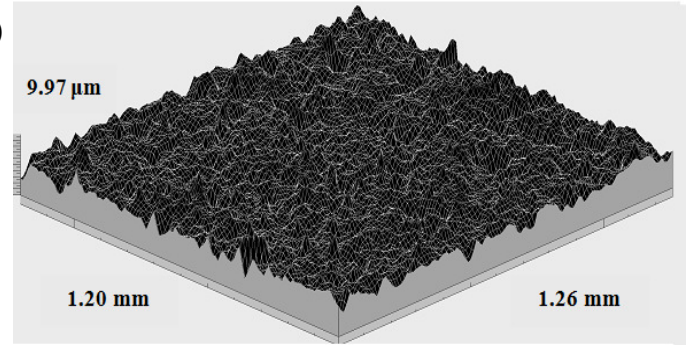

b)

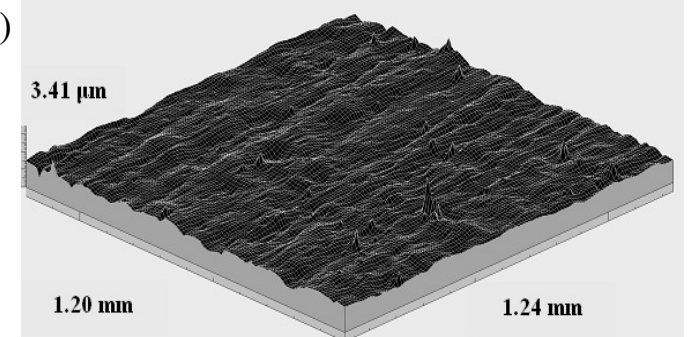

Fig. 1. 3D surface image of the sample with Ti-TiN coating (a) and without coating (b) [17]

Table 1

3D-texture parameters for the coated and uncoated Incoloy 800 samples [17]

\begin{tabular}{|l|c|c|c|c|c|}
\hline Samples & $\boldsymbol{S a}, \boldsymbol{\mu m}$ & $\boldsymbol{S s} \boldsymbol{k}$ & $\boldsymbol{S t r}$ & $\boldsymbol{V m c}, \mathbf{m m}^{\mathbf{3}} \cdot \mathbf{m m}^{-\mathbf{2}}$ & $\boldsymbol{S f d}$ \\
\hline Incoloy 800 & 0.116 & 0.140 & 0.125 & $1.02 \mathrm{E}-03$ & 2.33 \\
\hline Incoloy 800 with Ti-TiN coating & 0.659 & -0.679 & 0.701 & $4.00 \mathrm{E}-03$ & 2.42 \\
\hline
\end{tabular}

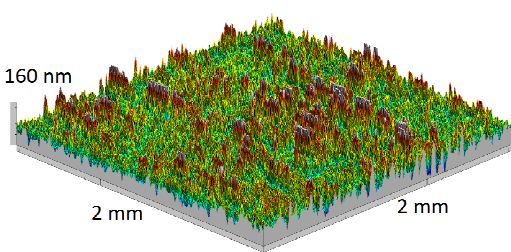

a)

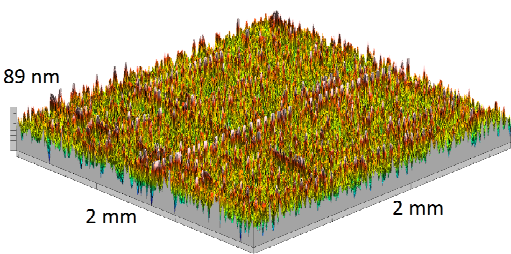

b)

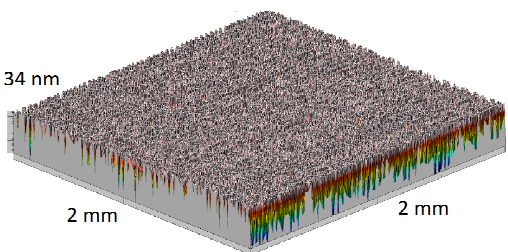

c)

Fig. 2. 3D surface image of the sample with a-CuC coating (a), with $\mathrm{Cu}$ coating (b) and without coating (c)

3D-texture parameters for the coated and uncoated 100Cr6 samples

\begin{tabular}{|l|c|c|c|c|c|}
\hline Samples & $\boldsymbol{S a}, \mathbf{n m}$ & $\boldsymbol{S s} \boldsymbol{k}$ & $\boldsymbol{S t r}$ & $\boldsymbol{V m c}, \mathbf{m m}^{\mathbf{3}} \cdot \mathbf{m m}^{-\mathbf{2}}$ & $\boldsymbol{S f \boldsymbol { d }}$ \\
\hline 100Cr6 & 6.2 & -1.260 & 0.500 & $1.38 \mathrm{E}-05$ & 2.81 \\
\hline 100Cr6 with Cu coating & 10.8 & 0.231 & 0.590 & $2.90 \mathrm{E}-05$ & 2.70 \\
\hline 100Cr6 with a-CuC coating & 18.3 & 0.791 & 0.115 & $4.23 \mathrm{E}-05$ & 2.61 \\
\hline
\end{tabular}

All coated samples have a higher 3D-texture amplitude parameter $S a$ value than the substrate on which it is coated. From this parameter alone, one could propose worse tribology properties (higher friction coefficient COF) for the coated surfaces, but in reality, tribology tests, conducted with a ballon-disc type tribometer, prove this consumption wrong. The average value of COF for the uncoated Incoloy 800 sample is $\mathrm{COF}=0.27$, while for the Ti-TiN coated sample, it is $\mathrm{COF}=0.24$. For the $100 \mathrm{Cr} 6$ sample $\mathrm{COF}=0.34, \mathrm{Cu}$ coating $\mathrm{COF}=0.70$ and $\mathrm{a}-\mathrm{CuC}$ coating $\mathrm{COF}=0.19$. The results show 
that $\mathrm{COF}$ can both increase and decrease, if $S a$ increases, suggesting that $\mathrm{Sa}$ alone is a pure surface description parameter.

Parameter $S s k$ can have a positive and negative value. Negative skewness confirms good lubricant retention ability, which is important, if additional lubricant is added on the surface. Unfortunately, in many cases, including the above-shown sample 100Cr6, Ssk parameter increases, if the coating is applied, thus reducing lubrication possibility.

Parameter Str defines texture isotropy, which defines whether the surface will have similar tribology properties in all sliding directions. Typically after applying the coating, the surface gets more isotropic than it was originally, especially when the coating thickness is significant ( $>2 \mu \mathrm{m}$ ). If the coating is thin, the substrate texture still dominates in the final texture. Some coatings, like a-CuC, can develop anisotropic texture due to its formation specifics.

High values of the hybrid parameter $S f d$ (close to 3 - maximum value of $S f d$ ) confirm complex and random character for all shown surfaces. The higher value of the functional parameter $V m c$ for all coated samples predicts a better bearing ability of the surface due to greater core material volume in contact during normal exploitation time (after wear-in).

Metrological research shows that a sample coated with a tough Ti-TiN layer has a set of surface texture parameters, which predicts that the coated sample is more suitable for tribological application in comparison with the uncoated one. While for the relatively soft $\mathrm{Cu}$ and a-CuC coatings, this is not that obvious. This indicates that one must take into account also the coating material properties, which in many cases might have a larger influence on tribology properties than the texture parameters. Thus, in [18] the authors proposed a new methodology of service life prediction of wear parts using 3Dtexture parameters for the elastic contact case, as well as for the elastic-plastic contact [19]. The aim was to propose a methodology for estimation of the service time of mechanical components using the technologically and metrologically controllable parameters, including 3D-texture parameters as input data for the equation. It is worth mentioning that the 3D- texture parameters for estimation of the service life of components were used for the first time. Such an approach ensures a fast and more precise estimation of the service time.

At this stage of the investigation, the set of the most appropriate 3D-texture parameters for the functional surface lifetime assessment includes the following parameters: $S a, S s k, S t r, V m c$, and $S f d$. Future research will be conducted, and currently chosen set off parameters might be modified in a way to be more suitable for various multifunctional nanostructured coating applications.

\section{Conclusions}

1. So far, clear assessment of using and understanding 3D surface texture parameters for wearresistant coatings does not exist;

2. The set of the most appropriate 3D-texture parameters for functional surface characterization ( $\mathrm{Sa}$, Ssk, Str, Vmc, and $S f d$ ) is offered;

3. 3D-texture parameters of functional surfaces are the most appropriate to match them with the tribological performance of the deposited coatings, and they can be used as feedback information to adjust the coating deposition technology to obtain the most promising texture parameters. The assurance of the desired 3D-texture parameters will allow us to assess the wear resistance of the coating with higher accuracy and to predict precisely the lifetime of the product.

\section{Acknowledgments}

This research is funded by the Latvian Council of Science, project "Carbon-rich self-healing multifunctional nanostructured smart coatings (NSC) for high-tech applications using high-power confined plasma technology for their deposition", project No. 2019/1-0385.

\section{References}

[1] Springis G., Rudzitis J., Leitans A., Avisane A. Wear calculation model for sliding surfaces with nano-coatings. Scientific Journal of Riga Technical University, series: Transport and Engineering, vol. 35, 2013, pp. 74-78. 
[2] Sedlaček M., Gregorčič P. and Podgornik B. Use of the roughness parameters Ssk and Sku to control friction-a method for designing surface texturing. Tibology Transactions, vol. 60, No. 2, 2017, pp. 260-266.

[3] Deleanu L., Georgescu C. and Suciu C. Comparison between 2D and 3D surface parameters for evaluating the quality of surfaces. Technologies in Machine Building, 2012, pp. 5-12.

[4] Das J., Linke B. Evaluation and systematic selection of significant multi-scale surface roughness parameters (SRPs) as process monitoring index. University of California, USA, 2017. 26 p.

[5] Bobrovskij I.N. How to select the most relevant roughness parameters of a surface: methodology research strategy. IOP Conf. Series: Materials Science and Engineering, 302, 2018. doi:10.1088/1757-899X/302/1/012066

[6] Wang T., Xie L.J., Wang X.B. and Shang T.Y. 2D and 3D milled surface roughness of high volume fraction $\mathrm{SiCp} / \mathrm{Al}$ composites. Defence Technology, 11, 2015, pp. 104-109.

[7] Librera E., Riva G., Safarzadeh H. and Previtali B. On the use of Areal Roughness Parameters to Assess Surface Quality in Laser Cutting of Stainless Steel with $\mathrm{CO}_{2}$ and Fiber Sources. Procedia CIRP 33, 2015, pp. 533-538.

[8] Zawada-Tomkiewicz A. Analysis of surface roughness parameters achieved by hard turning with the use of PCBN tools,. Estonian Journal of Engineering, 17, 2011, pp. 88-99.

[9] Zabala A., Blunt L., Tato W., Aginagalde A., Gomez X. and Llavori I. The use of areal surface topography characterization in relation to fatigue performance, Proceedings of 12th International Fatigue Congress FATIQUE 2018, vol. 165, 2018, 6 p.

[10] Myshkina N.K. and Grigorieva A.Ya. Roughness and texture concepts in tribology. Tribology in Industry, vol. 35, No. 2, 2013, pp. 97-103.

[11]Dzierwa A. Effects of surface preparation on friction and wear in dry sliding conditions. Tribologia, No. 2, 2017, pp. 25-31.

[12] Barányi I., Keresztes R., Szakál Z. and Kalácska G. Prediction of surface roughness parametres by new experimentally validated modelling algorithm under abrasive condition. Acta Polytechnica Hungarica, vol. 13, No. 7, 2016, pp. 197-208.

[13] Jansons E., Lungevics J. and Gross K.A. Surface roughness measure that best correlates to ease of sliding. Engineering for Rural Development, Jelgava, Latvia, 2016, pp. 687-695.

[14]Żak K. and Grzesik W. Metrological aspects of surface topographies produced by different machining operations regarding their potential functionality. Metrol. Meas. Syst., vol. 24, No. 2, 2017, pp. 325-335.

[15] ISO 25178-2 "Geometrical product specifications (GPS). - Surface texture. Areal - Part 2: Terms, definitions and surface texture parameters"

[16] Todhunter L.D., Leach R.K., Lawes S.D.A. and Blateyron F. Industrial survey of ISO surface texture parameters. CIRP Journal of Manufacturing Science and Technology, vol. 19, 2017, pp. 84-92.

[17] Urbahs A., Rudzitis J., Savkovs K., Urbaha M., Boiko I., Leitans A. and Lungevics J. Titanium Compound Erosion-resistant Nano-coatings. Key Engineering Materials, vol. 674, 2016, pp. 283288.

[18] Linins O., Krizbergs J. and Boiko I. Wear estimation using 3D surface roughness parameters. Key Engineering Materials, Engineering Materials and Tribology, vol. 527, 2013, pp. 167-172.

[19] Linins O., Jansons E., Leitans A., Boiko I. and Lungevics J. Estimation of service life of mechanical engineering Ccomponents. Key Engineering Materials, No. 799, 2019, pp. 71-76. 